\title{
Industrial Symbiosis Waste Exchange Identification and Optimization
}

\author{
Danielle M. Curri \\ North Carolina State \\ University \\ dmcurri@ncsu.edu
}

\author{
Tarek N. Aziz \\ North Carolina State \\ University \\ tnaziz@ncsu.edu
}

\author{
John W. Baugh \\ North Carolina State \\ University \\ jwb@ncsu.edu
}

\author{
Jeremiah X. Johnson \\ North Carolina State \\ University \\ johns24@ncsu.edu
}

\begin{abstract}
Industrial symbiosis is the concept that waste from industrial processes can be diverted and then reused as inputs into co-located industrial entities. While research to date has identified successful examples of industrial symbiosis and characterized formation processes, little is known about how new ecoindustrial parks can be designed and their performance optimized. In this paper, we describe how industrial symbiosis can be modeled and optimized during the development phase to assist in the creation of eco-industrial parks. We present a database framework, waste exchange identification algorithm, and Python-based optimization system that generates a mixed-integer linear programming model to minimize the amount of non-recycled waste produced. We illustrate the functionality of the approach on three test cases that demonstrate increasing levels of complexity. The optimization model can also accommodate multiple objectives, allowing further exploration of the benefits of industrial symbiosis at the design stage.
\end{abstract}

\section{Introduction}

In a world of limited resources, there is a growing interest in finding more sustainable ways to use and dispose of materials. Industrial symbiosis is the concept that waste from industrial processes can be reused as inputs into other co-located industrial entities. As described by Chertow [1], "industrial symbiosis engages traditionally separate entities in a collective approach to competitive advantage involving physical exchange of materials, energy, water, and by-products." Industrial parks that utilize industrial symbiosis are referred to as eco-industrial parks. Eco-industrial parks can be defined as a community of businesses that share resources leading to a series of benefits for both the businesses and the local community [2]. The implementation of industrial symbiosis in eco-industrial parks has benefits such as economic development, pollution remediation, water savings, land savings, and greenhouse gas reductions [3]. The benefits of industrial symbiosis have driven an increased interest in designing and implementing such practices.

In this paper, we present an open-source optimization model that selects and sizes a combination of industries to participate in an ecoindustrial park waste exchange network, subject to a set of constraints (e.g., land area limitations). The model offers prospective developers and planners the ability to determine the optimal combinations of industries to achieve maximum waste reduction for a given site. The program identifies the waste exchange network and then optimizes the exchanges. A waste exchange results in waste reuse.

Motivation for the work comes from a prior research project in which recommendations were made for implementing industrial symbiosis in a new industrial park. Gaps in the current literature and tools for implementing industrial symbiosis sparked the development of this new computational approach.

The paper is organized as follows. Section 2 presents related work on industrial symbiosis in both practice and research. Section 3 outlines our methodology and our mathematical programming formulation. Section 4 presents three case studies with increasing levels of complexity to demonstrate our approach. We conclude in Section 5 with further observations about the work and future research directions.

\section{Related Work}

An early and robust example of industrial symbiosis is an industrial park located in Kalundborg, Denmark. The physical connections between colocated industries started forming in the 1970s as a result of limited ground water availability and regulations. It was around this time that exchanges began between many of the facilities. Some of the major participants included an oil refinery, pharmaceutical plant, and a power station [1]. In the 
1980s, the participants began realizing the environmental implications of the exchanges. Over 2.9 million tons of waste were exchanged in a year. Additionally, the water consumption was significantly decreased, and 5,000 homes received district heat from recycled waste heat. Kalundborg triggered an interest in seeing what could be achieved through industrial symbiosis, resulting in efforts to help establish and optimize eco-industrial parks.

Research in industrial symbiosis optimization has focused on three main areas: water networks, energy networks, and material sharing [4]. With respect to water networks, optimization may be performed by graph technology or mathematical programming optimization. Wang and Smith [5] utilized pinch technology to minimize the amount of water used in an industrial park, an approach that serves as the basis for many industrial symbiosis water network optimizations using graph technology. Boix et al. [6] use mixed-integer linear programming to minimize the freshwater consumption and the regenerated water flow rate by identifying a network of water streams. More recent work [4] also utilizes mathematical programming due to the inability of pinch-based methods to handle several contaminants. Ramos et al. [7] use multi-leader-follower game theory to optimize industrial water networks, which allows different objectives or motives to be considered, the introduction of an authority, and confidentiality to be addressed. The identification and optimization of water networks using industrial symbiosis has been the most widely studied out of the three categories [4]. As a result, water network models are more complex and established than energy network and material sharing industrial symbiosis models. Therefore, water network models are often used when exploring the implementation of other industrial symbiosis networks.

The application of industrial symbiosis to energy networks has not been widely researched. Similar to early water network industrial symbiosis optimization some work has explored using pinch analysis [4]. However, material exchanges face a set of constraints that are quite different from energy network optimization. Energy networks are often constrained by transport capacity (e.g., power transmission), whereas material exchanges are largely constrained by the suitability of material reuse.

Optimization of material exchanges is less common than water exchanges due to the wide variety of materials that can be exchanged. Material exchanges add an increased complexity of identifying the exchanges that can take place. In the literature, there are multiple methods for identifying industrial symbiosis connections. Boyle and Baetz [8] describe a prototype knowledge-based decision support system to determine the potential treatments needed to recycle waste materials. Their system uses a series of criteria and available treatments to identify matches however the authors note several limitations to their system including the handling of metals, the number of tested treatments, reactions occurring due to treatments, and practical applications. Kincaid [9] describes the Industrial Ecosystem Development Project, a North Carolina study that gathered information from 182 industries regarding their inputs and outputs to identify industrial symbiosis connections. The information was gathered using a series of surveys, phone calls, and inperson interviews. A dataset was then built using the collected information and used to identify potential exchanges. Follow-up phone calls and meetings were used to gain detailed specifications regarding the materials. Trokanas et al. [10] use a semantic approach for input and output matches utilizing knowledge models and ontologies. Their approach uses a series of characterizations and calculations to find a fuzzy weighted average value that represents the similarity between inputs and outputs. Additionally, online tools have been used to connect companies to create industrial symbiosis through free-market mechanisms [11]. Other methods have included creating social network platforms to identify industrial symbiosis and creating knowledge repositories of known exchanges [12].

Once potential exchanges are identified, material optimizations can take place. While material sharing is common in eco-industrial parks, there are no widely adopted models to optimize material networks for industrial symbiosis [4]. Most available research is also limited to a single type of resource optimization [13]. Connelly and Koshland [14] present one of the first material exchange models, which utilizes a thermodynamic interpretation of ecosystem evolution and the concept of exergy. Cimren et al. [15] use a mixed integer linear program with a graphical user interface to minimize costs and environmental impacts. More recently, Nouinou et al. [16] utilize a bi-objective mathematical model to optimize for industrial symbiosis. The first objective is to maximize the amount of flow exchanges. The second is to maximize the total economic gain of the industrial park. Again, this work utilizes pre-defined industrial symbiosis connections. Alfaro and Miller create an optimization model to maximize the salary carrying capacity of a community development project in Liberia [17].They use preidentified exchanges to apply the model to a small farm.

With respect to our own work, the optimization model developed in this study arose from an earlier research effort in which we supported the development 
of a greenfield eco-industrial park. In the given context, the available tools and existing literature were found to be inadequate for the project. For instance, when designing a new eco-industrial park with industrial symbiosis, the industry material flows and sizes are often unknown. Available optimization tools are limited since they use set sizes of industries and predefined exchanges rather than determining ones that optimize performance. If the goal of the development is to achieve industrial symbiosis, these choice and size of industry dictate the potential exchanges that can take place. Once the possible connections have been identified, available literature fails to produce an easily adaptable optimization framework to explore various objectives. Additionally, many of the optimizations are custom made to specific industrial scenarios which is not easily translatable to other situations [12]. Finally, there is not an open-source, easily adaptable model that can be used to explore different materials sharing options while optimizing the connections.

\section{Methodology}

In this section we describe an optimization approach that identifies the waste exchange network and then optimizes the exchanges. To do this, we first identify all the combinations of material outputs (i.e., waste) that would be suitable to be inputs (i.e., feedstock) for other industries. These combinations, which we term matches, are found through a characterization protocol that considers a wide range of material attributes.

Intuitively, the approach should meet certain requirements to give a robust solution. For a given set of possible industries with an associated set of possible waste exchanges, it must determine which industries to include and size them within the feasible range allowed. Additionally, it must be able to address more complex situations such as selecting participants in a waste exchange when multiple matches for the same input or output are possible. For example, if one input has two output matches, it must decide to satisfy the input with a single output or with portions of multiple outputs.

As the eco-park increases in total size and more industries are considered, the problem becomes increasingly challenging: the objective function must ensure that while optimizing the connections, the overall waste of the park is minimized. In some cases, the park might be able to produce less waste by selecting industries that are not involved in a given set of matches. Prior work, such as that of Nouinou et al. [16], tends to sidestep the problem by simply optimizing the amount of exchanged waste. For example, in cases where one output has a particularly heavy waste stream and can be exchanged with multiple inputs, the total waste produced by the park may increase by including the stream for its connections rather than excluding it. We explore the issue further in Case Study 3.

\subsection{Notation}

We use the notation below in the models that follow:

$\mathrm{n}=$ industry number

$\mathrm{i}=$ input industry, $\mathrm{n}$

$\mathrm{j}=$ input material reference number

$\mathrm{k}=$ output industry, $\mathrm{n}$

$\mathrm{q}=$ output material reference number

$I_{i, j}=$ material input needed for industry $\mathrm{i}$, material $\mathrm{j}$

(kg)

$\mathrm{O}_{\mathrm{k}, \mathrm{q}}=$ material output produced from industry $\mathrm{k}$, material q $(\mathrm{kg})$

$\alpha_{\mathrm{i}, \mathrm{j}}=$ the input material intensity $\left(\mathrm{kg} / \mathrm{m}^{2}\right)$ for industry $\mathrm{i}$, input material $\mathrm{j}$

$\beta_{\mathrm{k}, \mathrm{q}}=$ the output material intensity $\left(\mathrm{kg} / \mathrm{m}^{2}\right)$ for

industry $\mathrm{k}$, output material $\mathrm{q}$

$A_{n}=$ the area for industry $n\left(m^{2}\right)$

$\mathrm{A}_{\mathrm{T}}=$ the total area available $\left(\mathrm{m}^{2}\right)$

$\mathrm{Y}_{\mathrm{n}}=$ industry binary indicator variable $(1=$ selected,

$0=$ not selected)

$l=$ a number that makes $Y_{n}$ equal to one when an exchange, $\varepsilon_{i, j}^{k, q}$ is positive

$m_{i, j}^{k, q}=$ the match from industry $\mathrm{k}$, output material $\mathrm{q}$

to industry $\mathrm{i}$, input material $\mathrm{j}$

$\varepsilon_{i, j}^{k, q}=$ the exchange from industry $\mathrm{k}$, output material $\mathrm{q}$ to industry $\mathrm{i}$, input material $\mathrm{j}(\mathrm{kg})$

$\mathrm{P}_{\mathrm{i}, \mathrm{j}}=$ primary material used for industry $\mathrm{i}$, input material $\mathrm{j}(\mathrm{kg})$ after waste-exchange connections are established in the system $(\mathrm{kg})$

$\mathrm{W}_{\mathrm{k}, \mathrm{q}}=$ total waste from industry $\mathrm{k}$, output material $\mathrm{q}$ after waste-exchange connections are established in the system $(\mathrm{kg})$

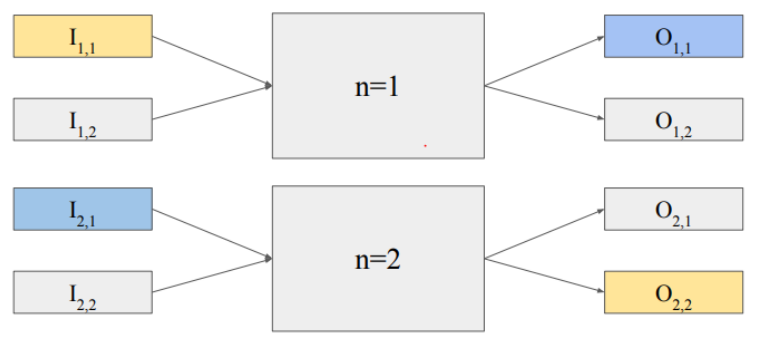


Figure 1. Illustrated notation applied to a two-industry system where each industry, $n$, has two inputs and two outputs.
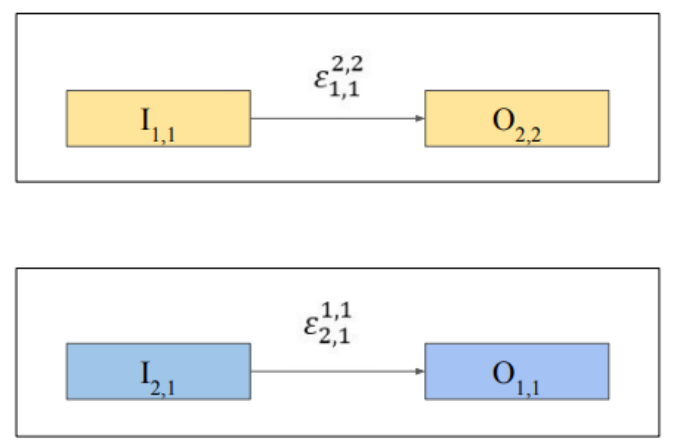

Figure 2. The two identified exchanges $\left(\varepsilon_{1,1}^{2,2}\right.$ ,$\varepsilon_{2,1}^{1,1}$ ) from the two-industry system shown in Figure 1.

\subsection{Matching Algorithm}

The first step is to identify all the potential industrial symbiosis connections. The connections are identified using a database csv file and a matching algorithm written in Python. The database is a representative data structure of the information needed to run the program. The database and program are designed to be flexible and can be adapted and scaled as information becomes available.

To run the matching algorithm and optimization system, the database requires an industry identification number; the minimum and maximum allowable area for the industry; material input names, intensities, and descriptors for the material characteristics; and material waste names, intensities, and descriptors for the material characteristics.

The matching algorithm utilizes this dataset to determine the viable matches for industrial symbiosis connections. We use input and output binary descriptors to characterize the inputs and outputs. Examples of the descriptors include: hazardous, nonhazardous, combustible, and non-combustible. For each of the descriptors, the material inputs and outputs are assigned a binary value based on the presence (1) or absence (0) of the given characteristic.

The matching program then uses the binary descriptors to define matches. For an output to be identified as a match to an input, the match must contain all the same true binary values (i.e., values of 1) as the input. The output can have additional true values but must satisfy the requirements of the input. This provision is to recognize that some of the outputs must undergo a transformation in order to be used as an input or the output may have an additional quality that does not hinder it from being used as the input.

In Figure 3, three binary descriptors (X, Y, and Z) are used to characterize the inputs and outputs. The three inputs and outputs have each been assigned binary descriptors. Based on the binary descriptors, an industrial symbiosis connection would be identified between $\mathrm{I}_{1,1}$ and $\mathrm{O}_{1,1}$. Additionally, a match between $\mathrm{I}_{1,1}$ and $\mathrm{O}_{2,1}$ is identified. $\mathrm{O}_{2,1}$ is identified as a match because it meets the requirements of input $I_{1,1}$ of having characteristics $\mathrm{X}$ and $\mathrm{Z}$ despite having an additional characteristic $\mathrm{Y}$.

\begin{tabular}{|c|c|c|c|}
\hline & $\mathbf{X}$ & $\mathbf{Y}$ & $\mathbf{Z}$ \\
\hline $\mathbf{I}_{1,1}$ & $(1)$ & 0 & $(1)$ \\
\hline & & & \\
\hline $\mathbf{O}_{1,1}$ & $(1)$ & 0 & $(1)$ \\
\hline & & & \\
\hline $\mathbf{O}_{2,1}$ & $(1)$ & 1 & $(1)$ \\
\hline
\end{tabular}

Figure 3. Example binary descriptors for three inputs and outputs.

\subsection{Model Formulation}

After all potential matches are identified, we then optimize the selection and sizing of the participating industries to achieve the desired environmental outcome. The optimization selects efficient exchanges. In this paper, we present a formulation that minimizes waste production that can also be adapted to minimize virgin input consumption. The model generator, written in Python and solved using Gurobi Optimizer [18], follows the formulation detailed in the following sections. Optimal industry selection is subject to the minimum and maximum area constraints for each industry, material input and output intensities, input and output binary descriptors, and other characterizations associated with each industry. For the formulation that we present here, the decision variables are the areas for each industry, the exchanges of material between industries, and the industry binary indicator variables.

This method that we describe, however, can be readily adapted for other environmental or economic objectives. Alternative objective functions include minimizing greenhouse gas emissions, energy use, water consumption, or system cost. These alternative objective functions would require altering the relevant intensity factors.

3.3.1. Objective Function. In this formulation, we define an objective function that minimizes the amount of non-recycled waste produced by the 
industrial park by utilizing the identified industrial symbiosis connections, as shown below:

$$
\operatorname{Min}\left(\sum W_{k, q}\right) \forall \mathrm{k}, \mathrm{q}
$$

Equation 2 determines the non-recycled waste $\mathrm{W}$ from industry k, for each material output (defined by output reference $\mathrm{q}$ ), as the difference between waste generated $\left(O_{k, q}\right)$ and successful waste exchanges occurring.

$$
W_{k, q}=O_{k, q}-\sum_{i=1}^{i} \sum_{j=1}^{j} \varepsilon_{i, j}^{k, q} \quad \forall i, j \in m_{i, j}^{k, q}
$$

The waste generated (before recycling) is calculated using Equation 3, which is determined by the waste intensity $\beta_{k, q}$ multiplied by the area of the industry $A_{n}$.

$$
O_{k, q}=\beta_{k, q} A_{n} \quad \forall k, q \forall \mathrm{n}
$$

3.3.2. Constraints. The model is subject to a series of constraints related to individual and cumulative sizing of the industries, non-negativity constraints, and conservation of mass.

Equation 4 ensures that the total area used by the selected industries equals the available area.

$$
A_{T}=\sum_{n=1}^{n} A_{n}
$$

In Equation 5, we constrain the individual areas of the industries to ensure that they can feasibly be built. The industries' minimum and maximum areas are predefined based on a reasonable range for the industries' viability. The industry areas can also take on the value of zero so that they can be excluded from the optimal solution. An industry binary indicator variable $Y_{n}$ is added so that the industry can take on the value of zero when it is not selected.

$A_{n}=\left\{\begin{array}{c}A_{n}=0 \\ Y_{n} A_{\min , n} \leq A_{n} \leq Y_{n} A_{\max , n}\end{array} \quad \forall A_{n}, Y_{n}\right.$

To ensure that the area can take on a value of zero and one, Equation 6 is added. In the constraint, $l$ is a number that makes $Y_{n}$ equal to one when an exchange, $\varepsilon_{i, j}^{k, q}$ is positive. The value of $l$ is found by multiplying the largest intensity by the total area to ensure that no exchange is greater than the value of $l$.

$$
l * Y_{n} \geq \varepsilon_{i, j}^{k, q} \forall \varepsilon_{i, j}^{k, q}
$$

Equation 7 states that the primary material (i.e., material sourced from outside the industrial park) used by input industry $i$, input reference number $\mathrm{j}$ must be non-negative.

$$
P_{i, j} \geq 0 \forall i, j
$$

Equation 8 determines the primary material $\mathrm{P}$ from industry $i$, for each material input (defined by input reference $j$ ) as the difference between waste generated $I_{i, j}$ and successful waste exchanges occurring.

$$
P_{i, j}=I_{i, j}-\sum_{k=1}^{k} \sum_{q=1}^{q} \varepsilon_{i, j}^{k, q} \forall k, q \in m_{i, j}^{k, q}
$$

The primary material before recycling is determined by Equation 9, where the input intensity $\alpha_{i, j}$ is multiplied by the area for the industry $A_{n}$.

$$
I_{i, j}=\alpha_{i, j} A_{n} \forall i, j \forall \mathrm{n}
$$

We also define a non-negativity constraint for the waste produced by industry $k$, output reference number $\mathrm{q}$ in Equation 10. The amount of waste exchanged, $\varepsilon_{i, j}^{k, q}$, also has a nonnegativity constraint as shown in Equation 11.

$$
\begin{gathered}
W_{k, q} \geq 0 \forall k, q \\
\varepsilon_{i, j}^{k, q} \geq 0 \forall i, j, k, q \in m_{i, j}^{k, q}
\end{gathered}
$$

3.3.3. Adaptation for multi-objective analysis. The model can also be adapted to consider additional objectives and develop trade-off curves. For the consideration of material flows, it is also important to consider the amount of virgin material required in addition to the amount of non-recycled waste being produced. The objective can be changed to minimize the amount of virgin primary material required:

$$
\operatorname{Min}\left(\sum P_{i, j}\right) \forall \mathrm{i}, \mathrm{j}
$$

$P_{i, j}$ is calculated as shown in Equation 8, and the constraints are held as described in section 3.3.2.

When considering environmental impact, it is helpful to understand the relationship between the amount of virgin primary material and the amount of non-recycled waste being produced. This can be explored by creating a trade-off curve where the amount of non-recycled waste being produced is on the $\mathrm{x}$-axis and the amount of virgin primary material is on the y-axis. The trade-off curve can also be used to look at additional implementations of industrial symbiosis besides the single-objective case. Since industrial symbiosis is a complex and interdisciplinary problem, the mathematically optimal solution may be less practical than other near optimal solutions. 
Therefore, it is important to present the decision maker with a range of solutions.

The trade-off curve is generated through a series of single-objective runs of the program. First, the program is run using the objective of minimizing the non-recycled waste. Using the calculated values for the areas and exchanges, the amount of new primary material needed can be found. The amount of nonrecycled waste being produced is the value of the objective function. These values are then recorded. Next, the program is run again with the objective function of minimizing the amount of virgin primary material required. An additional constraint is also added to the model to incrementally increase the amount of non-recycled waste produced:

$$
W_{k, q}>\rho * W_{k, q}^{\prime} \forall \mathrm{k}, \mathrm{q}
$$

$W_{k, q}^{\prime}$ is the value of the non-recycled waste produced in the previous run and $\rho$ is a numerical value used to incrementally increase the value of $W_{k, q}$. From the results, the amount of non-recycled waste being produced, and the amount of virgin primary material required are recorded. This step is repeated to gather points to generate the curve. The collected values of the amount of non-recycled waste being produced, and the amount of virgin primary material required are then graphed to form the trade-off curve.

\section{Case Studies}

Three case studies are presented to demonstrate the functionality of the program. For each case study, a database was formed using simulated data to demonstrate different features of the program. The databases were then input into the matching algorithm and optimization program. In the complete database, we propose a set of thirty-five descriptors to characterize industry inputs and outputs. However, this method is suitable for any number of waste characterizations as shown in the Case Studies which utilize three binary descriptors for simplicity.

The first case study is a simple case to demonstrate the basic function of the program. The first case study consists of two industries each with one identified input and one identified output as shown in Figure 4. Binary descriptors used to characterize the inputs and outputs and the input and output intensities are shown in Figure 4. While ideally all flows into and out of the system would be identified, we recognize that some flows may be difficult to characterize or may be so small they would not influence the optimization. These unidentified flows are shown in Figure 4 to demonstrate the mass balance. Additionally, the product leaving the system is shown in Figure 4.
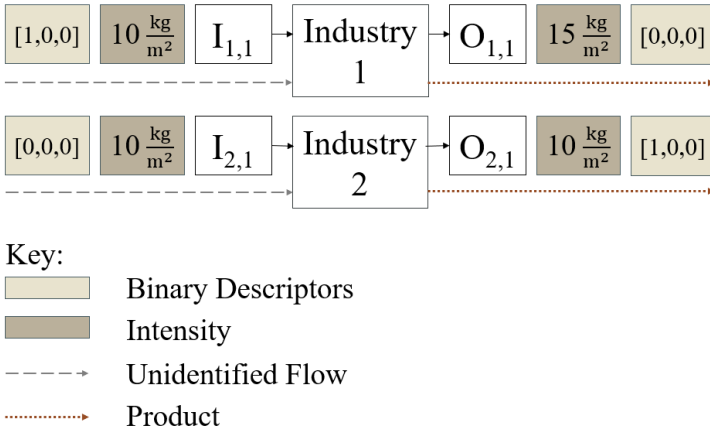

Figure 4. Assumptions for Case Study 1.

For this example, three descriptors are used to characterize the inputs and outputs. Industries 1 and 2 both have minimum areas of $25 \mathrm{~m}^{2}$ and maximum areas of $100 \mathrm{~m}^{2}$ and the site being developed has a maximum total area of $100 \mathrm{~m}^{2}$.

The optimization program gathers information from the database and inputs it into the matching algorithm. The matching algorithm then identifies the matches using the binary descriptors. As shown in Figure 4, $\mathrm{O}_{2,1}$ matches $\mathrm{I}_{1,1}$ because $\mathrm{O}_{2,1}$ meets the requirement of having a true binary value as the first binary descriptor set by $\mathrm{I}_{1,1}$. The identified match and database are then used as inputs to run the optimization program. The results of the optimization program are the sizes for the two industries and material flow for the identified match as shown in Figure 5. The optimization program sizes the two industries by scaling them to minimize the amount non-recycled waste. Due to their equivalent intensities for the input and output in the identified match, the two industries are both sized to $50 \mathrm{~m}^{2}$. The waste is minimized by recycling all $500 \mathrm{~kg}$ of waste produced by $\mathrm{O}_{2,1}$ to satisfy the $500 \mathrm{~kg}$ of input needed by $\mathrm{I}_{1,1}$. The total non-recycled identified waste produced by the park is the $750 \mathrm{~kg}$ of waste produced by $\mathrm{O}_{1,1}$. The amount of new material or identified source needed is the $250 \mathrm{~kg}$ of material required by $I_{2,1}$

The second case study exhibits more sophisticated decision-making capabilities of the program. For simplicity, the unidentified flows and products are not shown in Figures 6 and 7. In the second case study, four possible industries have been identified that can be used to fill a $100 \mathrm{~m}^{2}$ site. The allowable areas for Industries 1 through 4 are: (1) $20-40 \mathrm{~m}^{2}$; (2) $10-30 \mathrm{~m}^{2}$; (3) $5-40 \mathrm{~m}^{2}$; and (4) $1-100 \mathrm{~m}^{2}$. The binary descriptors and intensities are shown in Figure 6. 


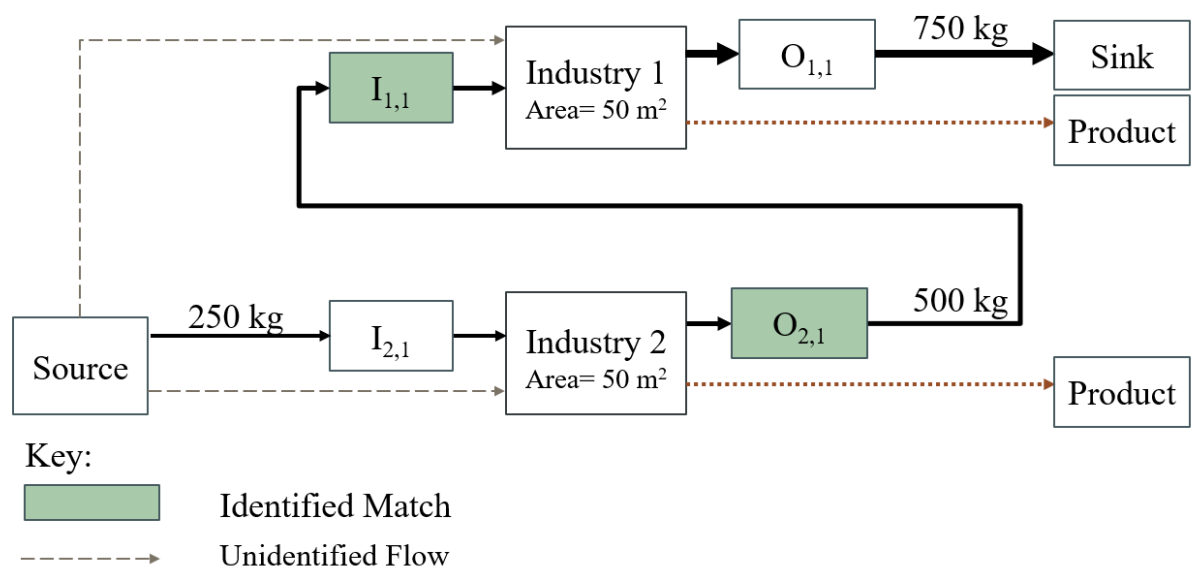

Figure 5. Optimal waste exchange for Case Study 1.

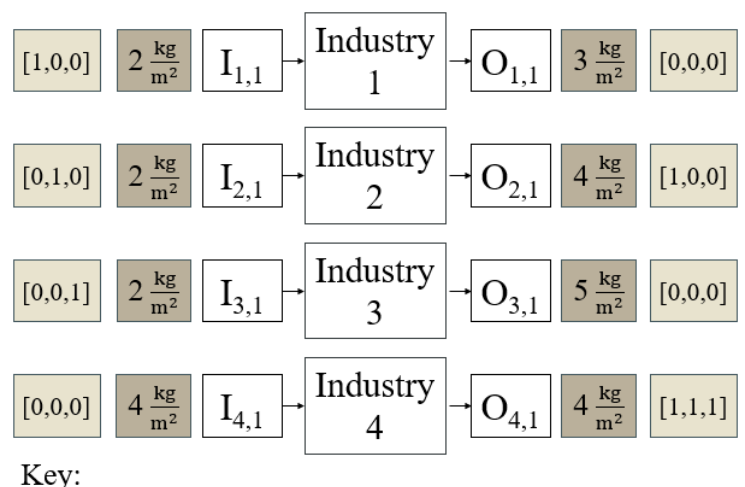

\begin{tabular}{|l}
\hline Key: \\
$\square \quad$ Binary Descriptors \\
\\
$\quad$ Intensity
\end{tabular}

Figure 6. Assumptions for Case Study 2.

The second case study is significantly more complex than the first scenario. The ranging minimum and maximum areas set limitations on the scaling for the industries. Additionally, the matching is more complex. Using the matching algorithm and binary descriptors, three matches are identified. Due to the true values for all three binary descriptors in $\mathrm{O}_{4,1}$, the program identifies that $\mathrm{O}_{4,1}$ matches $\mathrm{I}_{1,1}, \mathrm{I}_{2,1}$, and $\mathrm{I}_{3,1}$. Therefore, the optimization must decide how to use $\mathrm{O}_{4,1}$. The program can send the material to one input, split the material between inputs, send the material to none of the inputs, or exclude Industry 4 . The results of the optimization are shown in Figure 7. The optimization excludes Industry 3 , due to its high waste intensity. The optimization model then scales Industries 1 and 2 to take all the $\mathrm{O}_{4,1}$ it produces. The program decides to use Industries 1, 2, and 4 to minimize the non-recycled waste in the system.
The final case study demonstrates a scenario where using industrial symbiosis does not always produce the least amount of waste. When formulating the optimization model, multiple objective functions were considered. As described in the methods, the objective function minimizes the amount of non-recycled waste produced by the park. Rather than just maximizing the industrial symbiosis exchanges, the selected objective function recognizes cases where industrial symbiosis does not lead to the least amount of waste produced by the park. This scenario arises when an industry is particularly waste intensive or if a very clean industry is available. For example, in case study $3, \mathrm{O}_{1,1}$ has a heavy waste intensity and $\mathrm{O}_{4,1}$ has a relatively clean waste intensity relative to the other outputs as shown in Figure 8. 


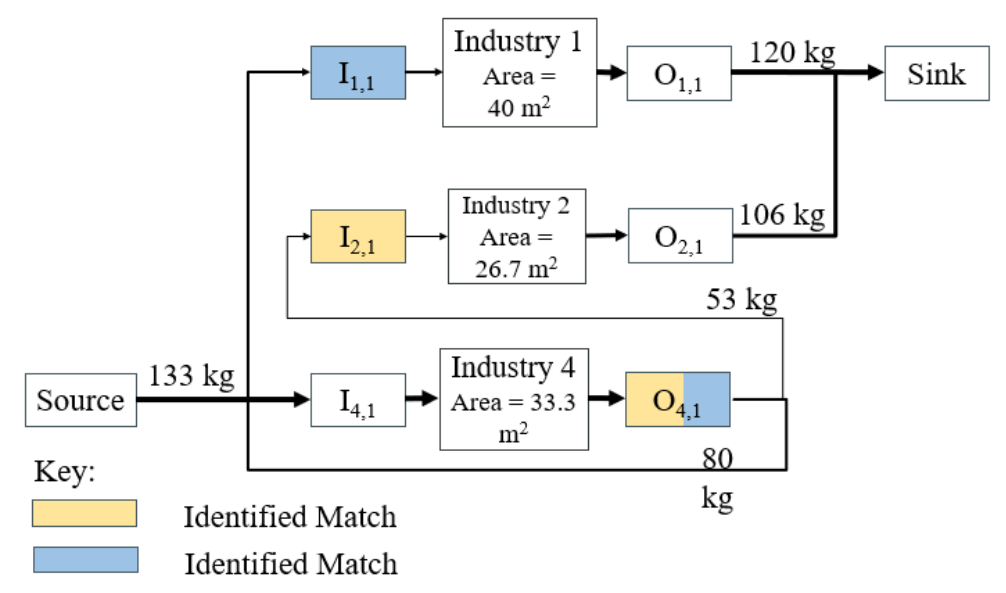

Figure 7: Optimal waste exchange for Case Study 2.

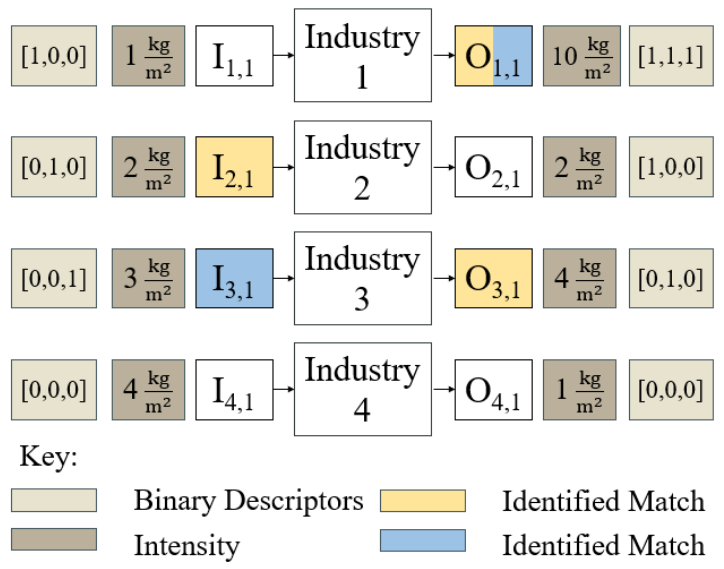

Figure 8. Assumptions for Case Study 3.

The final case study also demonstrates the ability of the program to generate trade-off curves to consider multiple objectives. As discussed in Section 3.3.3, the model can be retrofitted to calculate trade-off curves. By incrementing the amount of waste produced by the park, the relationship between the required material from the source and the material sent to the sink can be explored. When making decisions, this allows for considering both objectives of minimizing the amount of new material and minimizing the amount of wasted material.

For the case study, Industry 1 has a minimum area of $10 \mathrm{~m}^{2}$ and a maximum area of $30 \mathrm{~m}^{2}$. Industry 2 has a minimum area of $20 \mathrm{~m}^{2}$ and a maximum area of 40 $\mathrm{m}^{2}$. Industry 3 has a minimum area of $15 \mathrm{~m}^{2}$ and a maximum area of $40 \mathrm{~m}^{2}$. Industry 4 has a minimum area of $10 \mathrm{~m}^{2}$ and a maximum area of $80 \mathrm{~m}^{2}$. The binary descriptors and intensities are shown in Figure 8 . The site has an available area of $100 \mathrm{~m}^{2}$. The identified matches are displayed in Figure 8. The unidentified flows and products are not displayed in Figures 8 and 9.

The results of the optimization run are displayed in Figure 9. Industry 1 is excluded because, even though it can be exchanged with two inputs, the waste stream is so large that it does not lead to the optimal solution. While there are three identified matches, the system only utilizes the match of $\mathrm{O}_{3,1}$ to $\mathrm{I}_{2,1}$. Rather than including more exchanges, it is more advantageous to utilize the low waste intensity of $\mathrm{O}_{4,1}$. The system finds the optimal solution by scaling Industry 3 to meet the needs of Industry 2 and filling the remaining area with Industry 4.

For the third Case Study, a trade-off curve was generated to show the relationship between the inputs and the outputs, as shown in Figure 10. The trade-off curve was generated by running the program ten times. After the first run, the objective function was changed to minimize the amount of source needed. An additional constraint was added to ensure that that amount of waste produced was greater than the amount of waste produced in the previous run. For each trial, the amount of source needed, and amount of non-recycled waste produced was recorded. These points were then plotted with the amount of nonrecycled waste going to the sink on the $\mathrm{x}$-axis and the amount of source needed on the $y$-axis.

The optimal solution identified in the first run and shown in Figure 9 is the first point on the trade-off curve. While this solution minimizes the amount of non-recycled waste, it requires a large amount of new material from the source. By allowing for a small increase in the amount of non-recycled waste, the amount of new material required from the source generally decreases. For a user implementing 


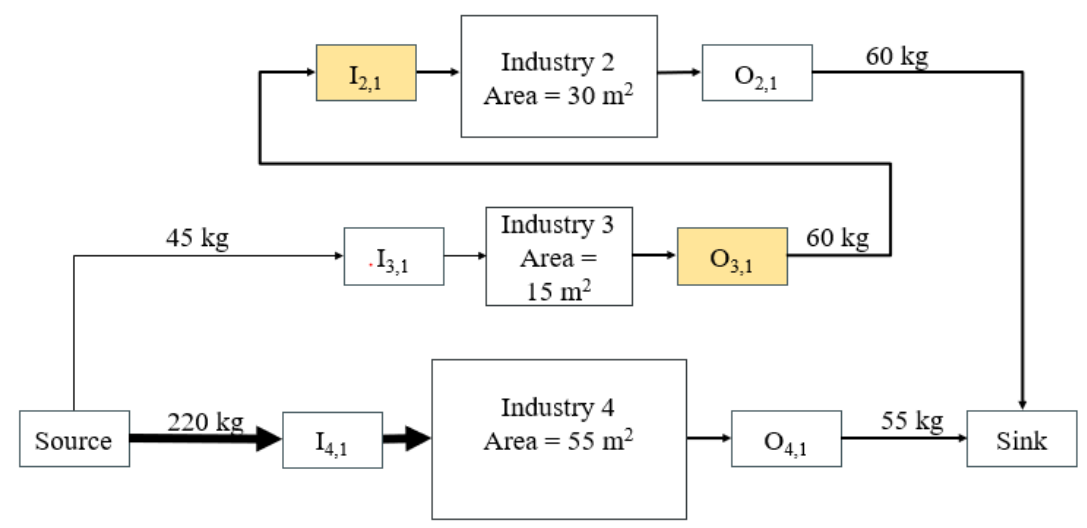

Key:

Identified Match

Figure 9. Optimal waste exchange for Case Study 3.

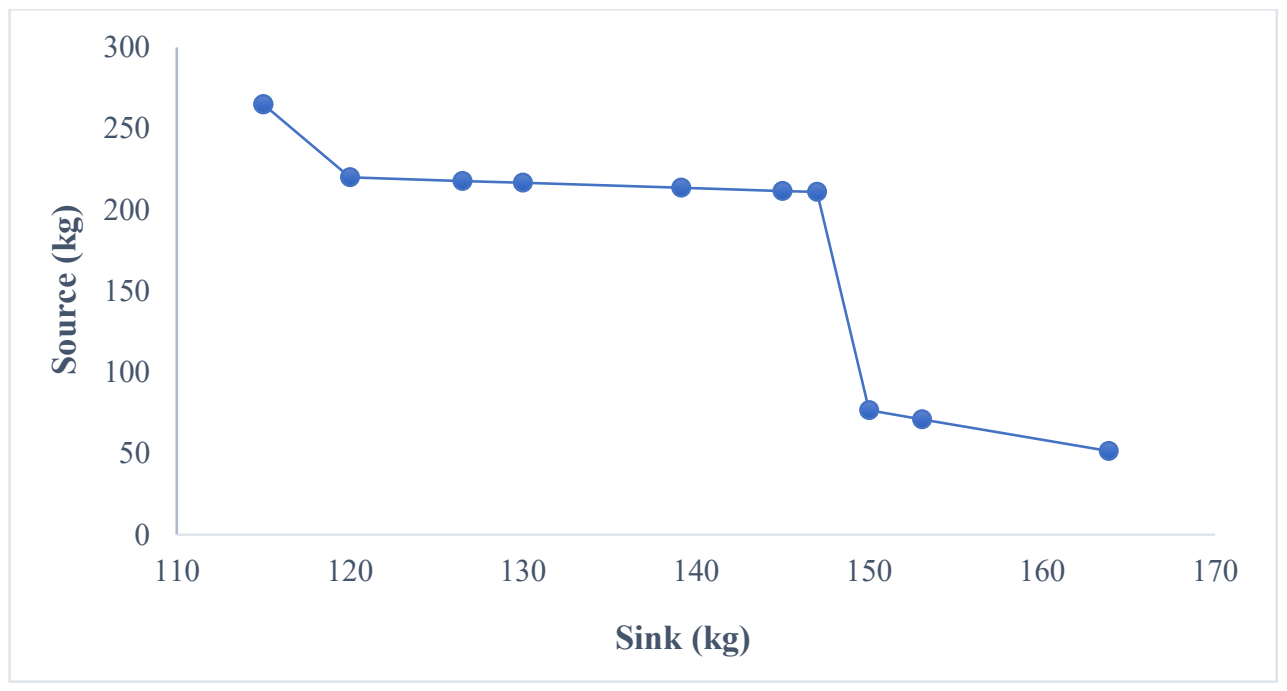

Figure 10: The trade-off curve between the use of primary material (source) and non-recycled waste (sink), shown for Case Study 3

industrial symbiosis, the trade-off curve assists in the decision-making process. If only the first run from the optimization is considered, the solution shown in Figure 9 would be selected. However, that is not necessarily the ideal solution when considering two key objectives: reducing the use of primary material and reducing non-recycled waste. By considering the trade-off curve, there is a large decrease in the amount of source needed when the source is approximately $75 \mathrm{~kg}$ and the sink is approximately $150 \mathrm{~kg}$. At this location, by allowing the system to produce more waste, the amount of source needed significantly decreases. This corresponds with Area 1 entering the system. While Area 1 has a large waste intensity, its waste can be exchanged with three different inputs. When Area 1 enters the system, its industrial symbiosis connections can be utilized resulting in a large decrease in the amount of new material needed.

\section{Conclusions}

In this paper, we presented a methodology to optimize and identify industrial symbiosis connections and demonstrated the capabilities of the program through three case studies. This program fills an important gap in the literature by establishing a more comprehensive methodology to identify and optimize 
greenfield industrial symbiosis through the selection, scaling, and matching of partner industries. While we demonstrated the ability of the program to establish industrial symbiosis by minimizing the amount of nonrecycled waste produced, the program can also be adapted to minimize the amount of primary material needed as shown in Case Study 3. Using these two objective functions, trade-off curves can be generated to assist in making decisions about industrial symbiosis. The model can also be easily adapted to explore additional objectives such as cost, water use, and greenhouse gas emissions by adapting the intensity factors and objective functions. This paper fills gaps in available literature by providing an adaptable framework to optimize the formation of industrial symbiosis in new industrial parks.

Future work can include further development of a multi-objective optimization framework to consider additional factors such as cost, water, and energy usage. Additionally, future work can explore using different intensity factors and objective functions such as greenhouse gas emissions. The model can also be utilized to explore patterns between identified optimal solutions that can be used to establish industrial symbiosis. Future work could also explore the expansion of our methods to real-world scenarios.

\section{References}

[1] M. R. Chertow, "I NDUSTRIAL S YMBIOSIS : Literature," Ind. Environ. Manag., 2000.

[2] P. C. on S. D. PCSD, "Eco-Industrial Park Workshop Proceedings," Eco-Industrial Park Workshop. 1996, [Online]. Available: https://clintonwhitehouse2 archives.gov/PCSD/Pu blications/Eco_Workshop.html\%0Ahttp://clinton2. nara.gov/PCSD/Publications/Eco_Workshop.html \#for.

[3] M. R. Chertow, “'Uncovering' industrial symbiosis," J. Ind. Ecol., vol. 11, no. 1, pp. 11-30, 2007, doi: 10.1162/jiec.2007.1110.

[4] M. Boix, L. Montastruc, C. Azzaro-Pantel, and S. Domenech, "Optimization methods applied to the design of eco-industrial parks: A literature review," J. Clean. Prod., vol. 87, no. 1, pp. 303317, 2015, doi: 10.1016/j.jclepro.2014.09.032.

[5] Y. P. Wang and R. Smith, "Wastewater minimisation," Chem. Eng. Sci., vol. 49, no. 7, pp. 981-1006, 1994, doi: 10.1016/00092509(94)80006-5.

[6] M. Boix, L. Montastruc, L. Pibouleau, C. AzzaroPantel, and S. Domenech, "Industrial water management by multiobjective optimization: From individual to collective solution through ecoindustrial parks," J. Clean. Prod., vol. 22, no. 1, pp. 85-97, 2012, doi:

10.1016/j.jclepro.2011.09.011.
[7] M. A. Ramos, M. Boix, D. Aussel, L. Montastruc, and S. Domenech, "Water integration in ecoindustrial parks using a multi-leader-follower approach," Comput. Chem. Eng., vol. 87, pp. 190 207, 2016, doi:

10.1016/j.compchemeng.2016.01.005.

[8] C. A. Boyle and B. W. Baetz, "A prototype knowledge-based decision support system for industrial waste management: Part I. The decision support system," Waste Manag., vol. 18, no. 2, pp. 87-97, 1998, doi: 10.1016/S0956-053X(97)100253.

[9] J. Kincaid, "Industrial Ecosystem Development Project Report,” no. May, p. 98, 1999, [Online]. Available:

http://www.tjcog.org/docs/regplan/solidwst/ieprept .pdf.

[10] N. Trokanas, F. Cecelja, and T. Raafat, "Semantic input/output matching for waste processing in industrial symbiosis," Comput. Chem. Eng., vol. 66, pp. 259-268, 2014, doi: 10.1016/j.compchemeng.2014.02.010.

[11] United States Business Council for Sustainable Development, "Materials - US BCSD," Facilitating Company-To-Company Industrial Reuse Opportunities That Support the Culture Shift To A Circular, Closed-Loop Economy. 2017, [Online]. Available: http://usbcsd.org/materials/.

[12] G. van Capelleveen, C. Amrit, and D. M. Yazan, "A Literature Survey of Information Systems Facilitating the Identification of Industrial Symbiosis," 2018, pp. 155-169.

[13] Z. Yeo, D. Masi, J. S. C. Low, Y. T. Ng, P. S. Tan, and S. Barnes, "Tools for promoting industrial symbiosis: A systematic review," J. Ind. Ecol., vol. 23, no. 5, pp. 1087-1108, 2019, doi: 10.1111/jiec. 12846.

[14] L. Connelly and C. P. Koshland, "Exergy and industrial ecology_-Part 1: An exergy-based definition of consumption and a thermodynamic interpretation of ecosystem evolution," Exergy, An Int. J., vol. 1, no. 3, pp. 146-165, 2001, doi: 10.1016/s1164-0235(01)00021-8.

[15] E. Cimren, J. Fiksel, M. E. Posner, and K. Sikdar, "Material Flow Optimization in By-product Synergy Networks," J. Ind. Ecol., vol. 15, no. 2, pp. 315-332, 2011, doi: 10.1111/j.15309290.2010.00310.x.

[16] H. Nouinou, D. Roy, and S. Hennequin, "Mathematical modelling for flows optimization within an industrial symbiosis," IFACPapersOnLine, vol. 52, no. 13, pp. 1319-1324, 2019, doi: 10.1016/j.ifacol.2019.11.381.

[17] J. Alfaro and S. Miller, "Applying Industrial Symbiosis to Smallholder Farms: Modeling a Case Study in Liberia, West Africa," J. Ind. Ecol., vol. 18, no. 1, pp. 145-154, 2014, doi: 10.1111/jiec. 12077 .

[18] Gurobi, "Gurobi - The fastest solver." [Online]. Available: https://www.gurobi.com/. 